\title{
Chapter 14 \\ Blue Stragglers in Clusters and Integrated Spectral Properties of Stellar Populations
}

\author{
Yu Xin \& Licai Deng
}

\subsection{Introduction}

Evolutionary population synthesis (EPS) has been widely used as a powerful tool to study the stellar contents of galaxies. In essence, EPS compares the observed, integrated spectrum of a galaxy with a combination of spectra of simple stellar populations (SSPs; single-age, single-metallicity populations) of different ages and metallicities, and decomposes the complex stellar contents into SSPs of known ages and metallicities to infer the galaxy's star-formation history. Over the past two decades, much work has been done to improve the accuracy of EPS and SSP models in various contexts (e.g., [6, 7, 19, 34, 35, 36]).

Unfortunately, population synthesis models still suffer from a number of limitations. One is our poor understanding of some advanced single-star evolutionary phases, such as of supergiants and asymptotic giant branch stars [41], while a second is an absence in the models of the results of stellar interactions, such as the so-called "stragglers" formed through mass transfer in binaries or stellar collisions. Such stars are usually very bright and can strongly affect the integrated-light properties of the entire system. The potential uncertainties inherent to EPS caused by ignoring these components could be much larger than those still remaining and due to the variety of input physics among different models.

In this chapter, we focus on the second limitation to the standard SSP models. With the updated knowledge of stars, in particular on the physical properties of binary and collisional interactions that eventually create the exotic blue straggler stars (BSSs) discussed in previous chapters in this book, a more realistic prescription of stellar populations is now possible. The goal of this chapter is to present a new set of SSP models which include contributions from BSSs.

Yu Xin \& Licai Deng

National Astronomical Observatories, Chinese Academy of Sciences e-mail: xinyu@bao.ac. cn, licai@bao.ac.cn 

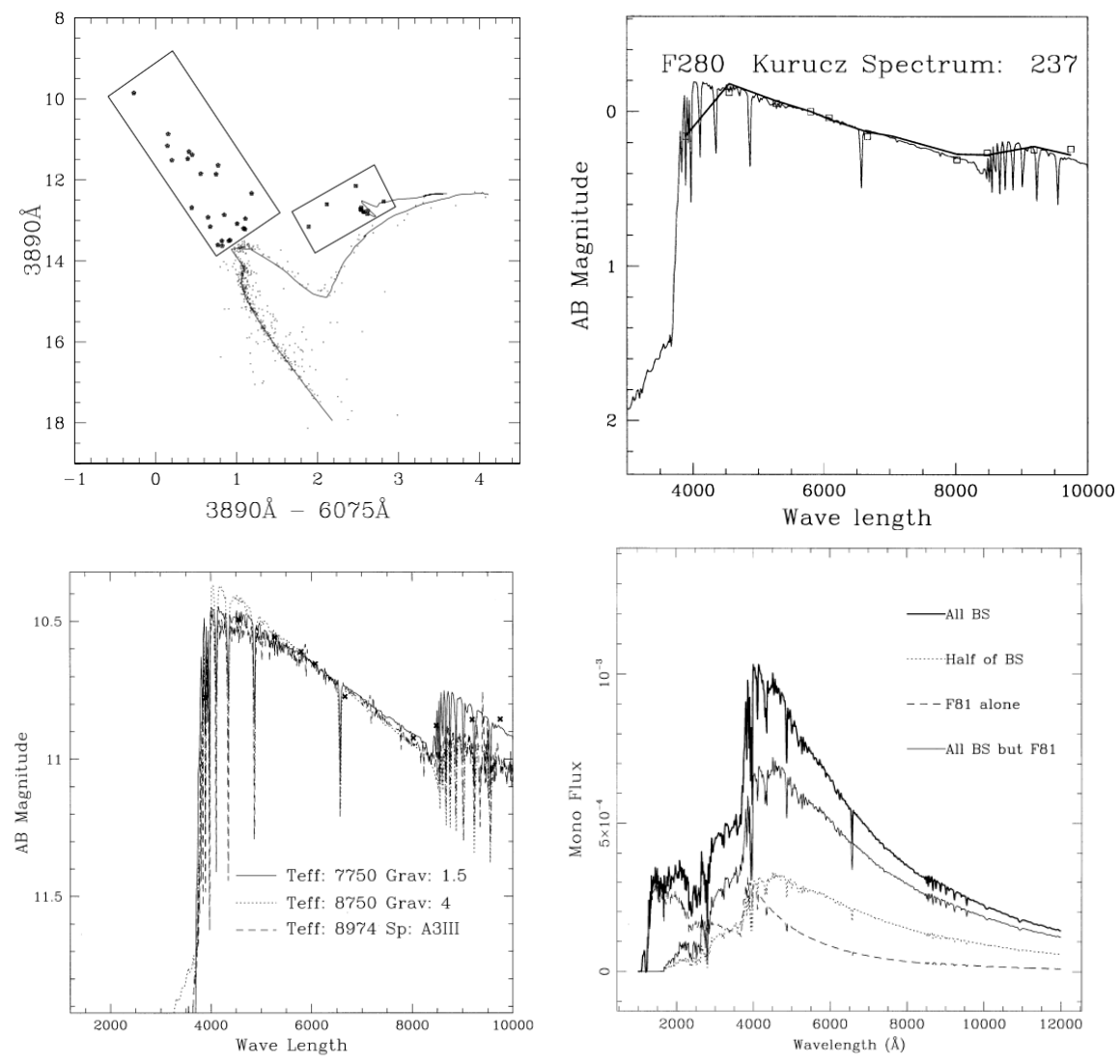

Fig. 14.1 This is an example of building up the integrated spectral energy distribution (ISED) of a star cluster based on accurate spectrophotometry from the BATC survey [13]. Upper left panel: CMD of M67 with all highly probable members, the left box along the extension of the main sequence marks the region of BSSs. Upper right panel: assigning a Kurucz spectrum to the star F280. In such a way we can actually determine the parameters of the stars (lower-left panel). Lower right panel: the ISEDs of the cluster with different combinations of spectral energy contribution to account for the stochastic effect.

BSSs are common and easily identified in colour-magnitude diagrams (CMDs) of star clusters. They are members of the host cluster and located above and blueward of the cluster's main-sequence (MS) turn-off. BSSs in a cluster are obviously the most luminous blue stars in the CMD (see the top-left panel of Fig. 14.1p, therefore they are the primary contributors in the total light of the cluster. The standard theory of single-star evolution cannot explain the presence of BSSs in SSPs' CMDs, and thus the standard SSP models do not include the contributions of BSSs. All currently accepted scenarios of BSS formation are related to stellar interactions. Coalescence in primordial binaries can launch BSSs to positions up to 2.5 magnitudes brighter than the MS turn-off [27, 9]. Mergers of binary-binary systems can produce possible 
BSSs with masses four times those of stars at the MS turn-off [21]. Given the high luminosities and common presence of BSSs in stellar systems (e.g., [1] for open clusters; [30] for globular clusters; [25] for dwarf galaxies), we believe that we must consider the effects of BSSs in studies of stellar populations using population synthesis in unresolved observations. The key issue is how to accurately include BSS contributions in SSP models.

Studies show that no single mechanism can account for the entire BSS population observed in any one star cluster [33]. This means that it is not easy to theoretically measure the respective contribution of BSSs in SSPs of different ages and metallicities. Although the detailed physical properties of BSSs can be better understood now (see Chap. 3, 8, 9, and 12), a comprehensive and robust model for BSS content in star clusters is still missing. Therefore, building up BSS population characteristics empirically from the statistics of a large sample of star clusters could be more practical and reliable than relying on incomplete theoretical approaches. In this way, the behaviour of BSSs (in terms of their specific frequency and relative distribution with respect to the MS turn-off in CMDs) can be modelled. Open clusters (OCs) in the Galaxy have a number of advantages for use as a working sample: (i)most have photometric data, thus enabling accurate determination of their fundamental parameters; (ii) many have multi-epoch proper motion and/or radial velocity data, so that their cluster membership probabilities can be measured accurately; and (iii) they are representative of the environments of stellar populations shortly after their birth. Compared to OCs, globular clusters (GCs) are usually much more massive and often contain multiple stellar populations (e.g., [29]), which renders them questionable as SSPs.

Preliminary modelling of BSS effects has been done on the basis of individual clusters in our previous papers [11, 37, 40, 39], where we (i) introduced the method used for calculating the realistic, integrated spectrum of a star cluster including the contribution of BSSs; (ii) analysed the modifications to the integrated spectra and the broad-band colours caused by BSSs; and (iii) estimated the possible uncertainties in the conventional SSP models, showing that the ages of star clusters can be underestimated by up to $50 \%$ if BSS contributions are not considered. To overcome such a great discrepancy, we present a set of BSS-SSP models based on a statistical study of galactic OCs. Our models cover the wavelength range from $91 \AA$ to 160 $\mu \mathrm{m}$, ages from 0.1 to $20 \mathrm{Gyr}$ and metallicities $Z=0.0004,0.004,0.008,0.02$ (solar metallicity) and 0.05 .

This chapter is organised as the follows. In Sec. 14.2, we demonstrate how BSSs can affect the integrated spectral properties of a star cluster, taking one of the most studied old clusters, M67, as an example. In Sec.14.3, we present the statistical results of the properties of BSS populations based on 100 Galactic OCs, supplemented by a few rich GCs in Magellanic clouds in Sec. 14.4. In Sec. 14.5, we describe the construction procedure of our models, as a generalised method based on that for M67, and we discuss our model results and compare them with those of Bruzual \& Charlot ([7], hereafter BC03). Finally, a summary, brief discussion and prospectives are presented in Sec. 14.6 


\subsection{M67: Setting up the Scheme}

We started to consider quantitatively the effects of BSS on the integrated spectral properties of star clusters, and to constrain the theoretical models of SSPs in 1999 using the well studied and relatively rich galactic OC, M67 [11].

Almost all bright stars in the cluster region (about 1 degree in diameter) have reliable membership information, which is very important to the argument we want to pursue. This cluster were observed using a 15 intermediate-band filter photometric system covering the whole optical wavelength range between 3000-10000 $\AA$ (BATC survey, [13]). Such an observation is ideally suited for our purpose. Figure 14.1 demonstrated the key steps to build the integrated spectral energy distribution (ISED) of the cluster:

- First of all, we need to find out the BSS members in the cluster. The upper left panel is a CMD of the cluster in two passbands (centred at $3890 \AA$ and $6075 \AA$, respectively), with the $x$-axis being the colour - defined as the difference between magnitudes in two magnitudes. All the BSSs confined by the left box at the upper bluer extension of the MS are almost sure members of M67.

- The upper right panel show how precisely we can anchor a Kurucz spectrum to the observed spectral energy distribution (SED) determined in all the filters for the famous BSS member F280 in M67. In fact, a BSS star may not be a single star, therefore it is not possible to have parameters that are defined for single stars, such as effective temperature and gravity. Thus, the spectrum assigned to the star this way is a "pseudo" but still constitutes an useful approximation. The spectrum assigned is indeed close enough and can represent the star's spectral property.

- In this way, we can then accurately derive its "physical parameters", given the metallicity is solar as measured by numerous other observations (presumably also treated as single stars). This is demonstrated in the lower left panel. In our work, we assumed that the BSSs can be taken as single stars in terms of spectral properties.

- The ISED of the cluster is obtained by summing up individual SED of all bright members. To account for possible stochastic effects due to the low number of BSSs, we plotted different combinations of BSS sample in M67 (lower right panel).

As a conclusion, the ISED of M67 built this way is apparently different from that of the theoretical SSP model corresponding to its age and metallicity. We further point out that, with the existence of BSSs, and when observed at unresolved conditions (such as in remote galaxies), the actual stellar population may appear substantially younger and/or more metal poor, sometimes less total mass compared to standard SSP models. M67 is just a typical old age cluster in our Galaxy, that can represent real stellar population of similar age and metallicity in any other galaxies, which means, the widely used conventional SSP models in EPS need to be modified in order to be correctly applied to the analysis of stellar content in galaxies. 
Thanks to the accurate but elaborate 15 intermediate band photometry [11], the technique to build the ISED of M67 based on photometric observations is very unique and reliable. However, BATC photometry is not available for the entire galactic OC sample. Other photometry, including broadband, can also be helpful. The methodology is defined in Sec. 14.5.2

\subsection{ISEDs of Galactic Open Clusters}

Building up a library of ISEDs of a large sample of star clusters is an observational approach towards realistic SSP models. Such a library can also be used to constrain theoretical efforts towards the same aim. By using the existing photometry and membership information of galactic star clusters, we have carried out a series of work in the past few years [37, 38, 39, 40].

Galactic OCs are the most studied stellar systems in the Galaxy, and have relatively more information about their physical properties including membership, age, metallicity, etc. However, the content of BSSs in OCs is rather stochastic due to the limited number of stars and BSSs, while all the BSS population may involve different ways of formation, and can be affected by dynamical processes in the cluster and with their environment.

In [37], a small sample of 27 Galactic OCs from Ahumada \& Lapasset ([1]; AL95 in the following) was selected to discuss the BSS contribution in a statistical way. The BSS population is better presented in the CMD of older OCs, thus the 27 OCs are all older than $1 \mathrm{Gyr}$, and provide a constraint to SSP models of the same age and metallicity range.

The basic parameters of the selected clusters are given in Table 1 of [37], where columns (1)-(3) give the cluster name, right ascension, and declination (J2000.0); columns (4)-(7) are the ages, colour excesses $[E(B-V)]$, distance moduli (DMs) and metallicities $(Z)$ of the clusters; the number of BSSs, $N_{\mathrm{BSS}}$, and $N_{2}$ values are listed in columns (8) and (9), respectively; and finally, column (10) is the reference number. Values of $N_{\mathrm{BSS}}$ and BSS photometric data for selected clusters are quoted directly from AL95. The $N_{2}$, defined as the number of stars within an interval of 2 magnitudes below the turn-off point for a given cluster (see detailed discussion in Sec. 14.5, is also from AL95. The other basic parameters of the selected clusters, specifically the age, $Z, E(B-V)$, and $\mathrm{DM}$, are extracted from the more recent photometric and theoretical work when results newer than AL95 are available.

$\mathrm{N}_{2}$ is regarded as a very important parameter that indicates the richness of the theoretical stellar component in the simple population synthesis scheme. For each cluster, this count is made in the same CMD from which the BSSs are selected. Then the ratio $N_{\mathrm{BSS}} / N_{2}$ can be taken as a specific BSS frequency in a cluster and can be used as a probe for the cluster internal dynamic processes concerning BSS formation.

It is worth emphasising here that not all the clusters have complete membership determinations in terms of both proper motion and radial velocity. The selection of 
BSS candidates in AL95 is basically according to where they appear in the observed CMDs. In our work, all the BSSs are treated equally, without any further detailed membership measurements and BSS identification.

In order to demonstrate the effects of BSSs on the conventional ISEDs in the case of old OCs, we construct the ISED of the normal star and the BSS population in one cluster separately. The steps of how we build the composite SSP models including BSS contribution will be presented in details in Sec.14.5. The results with the OC samples were rather stochastic due to the small number of BSSs, or because of the small total number of stars in a given cluster. The ISEDs vary from BSS dominant - one or a few very bright BSSs - or one in which BSSs contribute nothing to the ISED (see Fig. 4 in Xin, Deng \& Han [40], and discussions on statistics there. This remind us that rich star clusters should be used, or find a proper way to enhance statistics of the OC sample such as binning the clusters in parameter space (see Section 14.5.1.

\subsection{The Massive Star Clusters in the LMC \& SMC}

Star clusters in the Magellanic Clouds are massive and have a large span in age. Unlike the globular clusters in the Galaxy, they represent much better stellar populations in age coverage, and therefore serve as great targets for our work. In this section, the LMC cluster ESO 121-SC03 is analysed as the first example to detect BSS contributions to the conventional SSP models using a massive intermediate-age cluster in a low-metallicity environment. ESO 121-SC03 is a distant northern Large Magellanic Cloud (LMC) cluster, lying at a projected angular separation of 10 degrees from the LMC centre. It is described as a unique LMC cluster by Mackey, Payne \& Gilmore ([24]), because it is the only known cluster to lie in the LMC age gap. A significant number of previous studies (e.g., [6, 26, 24]) claim an absolute age for ESO 121-SC03 in the range of 8-10 Gyr. Mateo et al. [26] obtained $[\mathrm{Fe} / \mathrm{H}]=$ $-0.9 \pm 0.2$ combined with a reddening of $\mathrm{E}(B-V)=0.03$ mag. Mackey et al. [24]

derive $[\mathrm{Fe} / \mathrm{H}]=-0.97 \pm 0.01$ and $\mathrm{E}(\mathrm{V}-\mathrm{I})=0.04 \pm 0.02$ for the cluster. They also mark a region in the CMD used to define BSS candidates in the cluster, but they do not study the BSS population in detail.

As shown in Fig. 14.2, ESO 121-SC03 is a "clean" cluster and includes an obvious population of BSS candidates, which is very advantageous for our main aim of analysing the BSS contributions to the ISED of the cluster. This example demonstrated that, with statistics better than Galactic OCs, BSSs may have significant contributions to a stellar population [39]. Figure 14.3 shows the ISED of the cluster and how it is affected. With either BSSs only in the half-light radius $\left(R_{\mathrm{HL}}\right)$ or full sample in the cluster, apparent alternation due to BSSs to the theoretical ISED of its SSP model (the think solid line) is present. The change is stronger for the full sample, and the changes are always more prominent in the shorter wavelengths. This may actually infer either a younger age or lower metallicity when observed at unresolved conditions. 
Fig. 14.2 Cleaned CMD for ESO 121-SC03. The bestfitting Padova 2000 isochrone is overplotted. The corresponding fit parameters are included in the figure legend. In the CMD, we define a region that we will use for the identification of the cluster's BSS population. The ZAMS is the Padova 1994 isochrone at $\log \left(\right.$ age $\left.\mathrm{yr}^{-1}\right)=6.60$ and $[\mathrm{Fe} / \mathrm{H}]=-0.97 \mathrm{dex}$. The solid bullet is the cluster's MS turn-off. The solid triangles represent the MS ridge line of the cluster. Pentagons are BSSs. Pentagons with circles are BSSs inside the half-light radius $\left(R_{\mathrm{HL}}\right)$ of the cluster.

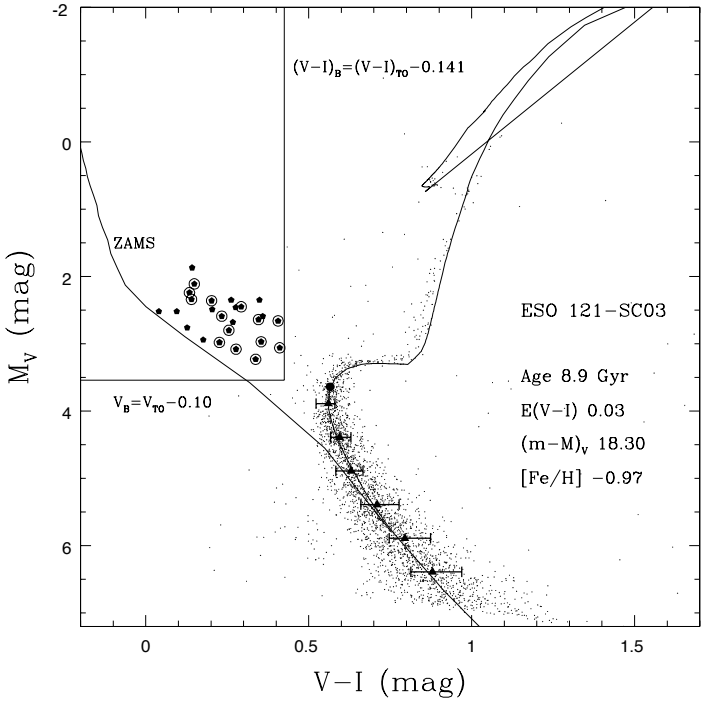

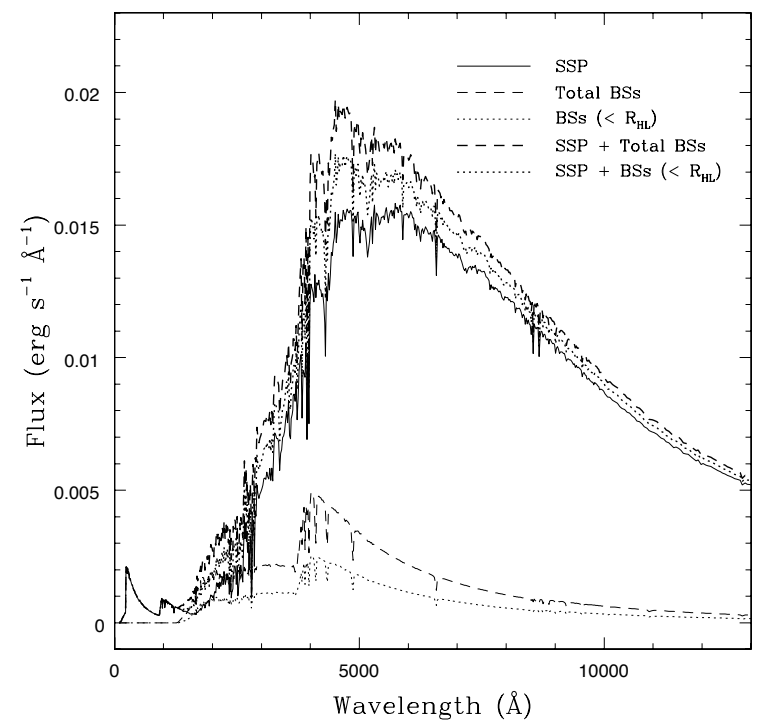

Fig. 14.3 ISED modifications. The BSS contributions are presented for two cases. The solid line is the ISED of the SSP component. The thin dotted line is the ISED of the BSS component for case (i): BSSs within $R_{\mathrm{HL}}$. The thin dashed line is the ISED of the BSS component for case (ii): all BSSs in the cluster. The heavy dotted line is the synthetic ISED of the SSP component and the case (i) BSS component. The heavy dashed line is the synthetic ISED of the SSP component and the case (ii) BSS component. 


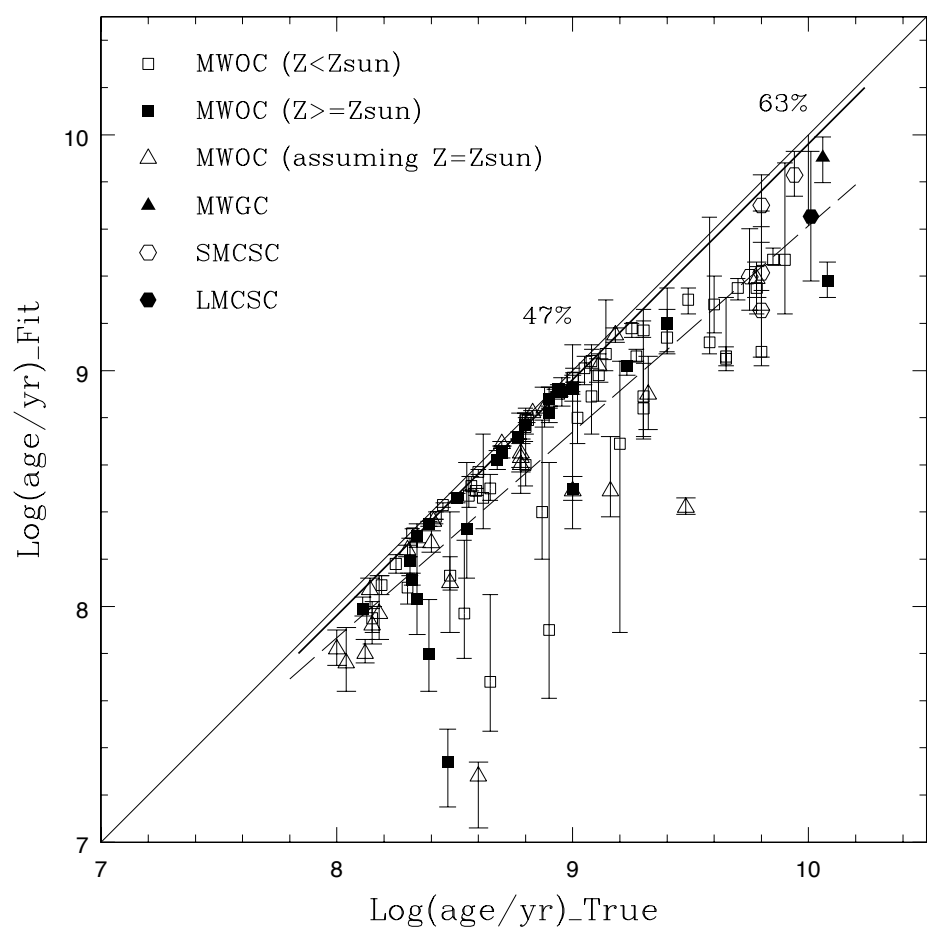

Fig. 14.4 The underestimation of age value by the conventional SSP models. The $x$-axis is the real age of star clusters, while the $y$-axis is the best-fitting age from the conventional SSP models. Different symbols mean different working samples. The ashed line is the least-square fitting of all the data points. The heavy solid line is given according to the formula of $t_{b}=\left(0.17+8.27 Z_{s}\right)+$ $\left(1.38-14.45 Z_{s}\right) t_{s}$ [22], assuming $50 \%$ binaries in stellar populations and using solar metallicity $\left(Z_{s}=0.02\right)$. Distances between the diagonal and the dashed line are measured to detect the age uncertainty - $\left(\right.$ age $_{\text {real }}-a_{\text {age }}$ fit $) /$ age $_{\text {real }}$. The underestimation of age value by the conventional SSP models becomes larger for older population. It is $47 \%$ at $1 \mathrm{Gyr}$, and $63 \%$ at $10 \mathrm{Gyr}$.

Figure 14.4 concludes our work on individual cluster's base. If we fit the ISED of star clusters by using the conventional SSP models, and we compare the ages of the cluster measured by observations of clusters (taking as true age, the horizontal axis), to that of the best fit SSP model (the vertical axis), it is very clear that the fitted ages are substantially younger than the true ages of the clusters for the whole sample of clusters (all types). Quantitatively, at the true age of $\sim 1 \mathrm{Gyr}$, the deviation is $47 \%$, and is $63 \%$ at the age of $\sim 10$ Gyr. This fact tells us that EPS may have seriously underestimated ages for populations within the age range covered by our sample.

Certainly, a larger sample of such rich clusters with good coverage in both age and metallicity is highly desired in order to constrain the method we proposed. Observational aspects, including detailed individual BSS, statistics of all BSSs in, and their dynamical effects on, rich GCs can be found in Chap. 5 . 


\subsection{Building Up an Empirical SSP Library}

In order to create empirically a library for use in EPS, a dataset of observed star clusters covering wide enough parameter space (age, metallicity) has been made available in our previous work. In this section, we are going to describe how the library is actually built.

\subsubsection{A General BSS Distribution Function in Stellar Populations}

Basically, two properties of BSS populations are relevant to the modelling of BSS behaviour in SSPs, i.e. the number of BSSs $\left(N_{\mathrm{BSS}}\right)$ in the SSP and their distribution in the SSP's CMD. In this paper, both properties are obtained empirically from the observed OCs' CMDs. A working sample including 100 Galactic OCs from the catalogue of AL95 is adopted to secure the reliability of the statistical results.

Figure 14.5 shows the differences among the number distributions of OCs containing BSSs versus age for the Ahumada \& Lapasset ([2], hereafter AL07) catalogue (horizontal hatching), the AL95 catalogue (slanted hatching from bottom left to top right) and our sample (slanted hatching from top left to bottom right). AL95 and AL07 published the most complete catalogues to date in terms of photometric data of BSSs in Galactic OCs. They include almost all OCs containing BSSs in the solar neighbourhood. Figure 14.5 shows that, compared to AL95, AL07 dramatically reduced the number of young OCs containing BSSs, mainly because of the difficulty of identifying BSSs in the CMD of a star cluster that does not exhibit at least a fully developed red giant branch (RGB) phase (particularly when information on membership probability is lacking). Moreover, most of the BSSs in young OCs (i.e., $\log ($ age/yr $)<8.0$ in this work) from both catalogues are located close to the MS turn-off point in the CMD, which means that it is hard to distinguish BSSs from MS stars. Such BSSs cannot effectively modify the spectral intensity of a cluster. Therefore, we start the selection of our working sample based on OCs with $\log ($ age $/$ yr $) \geq 8.0$.

Meanwhile, to construct a spectrum of the BSS population for a given OC, we need to obtain the physical parameters of the BSSs in the CMD of the OC. To do this, we need the age, metallicity, colour excess and distance modulus for each OC, which are not all included in either AL95 or AL07. Therefore, we decided to keep only the photometric data of BSSs from AL95, to ensure a homogeneous selection of the BSS sample. We collected the remaining OC parameters from the recent literature (see Table 1 in [40]). In practice, OCs with $\log ($ age/yr) $\geq 8.0$ from AL95 are included as sample clusters if reliable parameters can be found, in the sense that most of the BSSs are located at reasonable positions in the CMD with respect to the Padova 1994 isochrone for the OC's age and metallicity.

There are 33 OCs with age $\geq 1.0 \mathrm{Gyr}$ in the combined catalogue comprised of AL95, AL07 and our working sample. Older OCs have better statistics as regards their BSS populations. Using the photometric data of the BSSs in these 33 OCs as an 


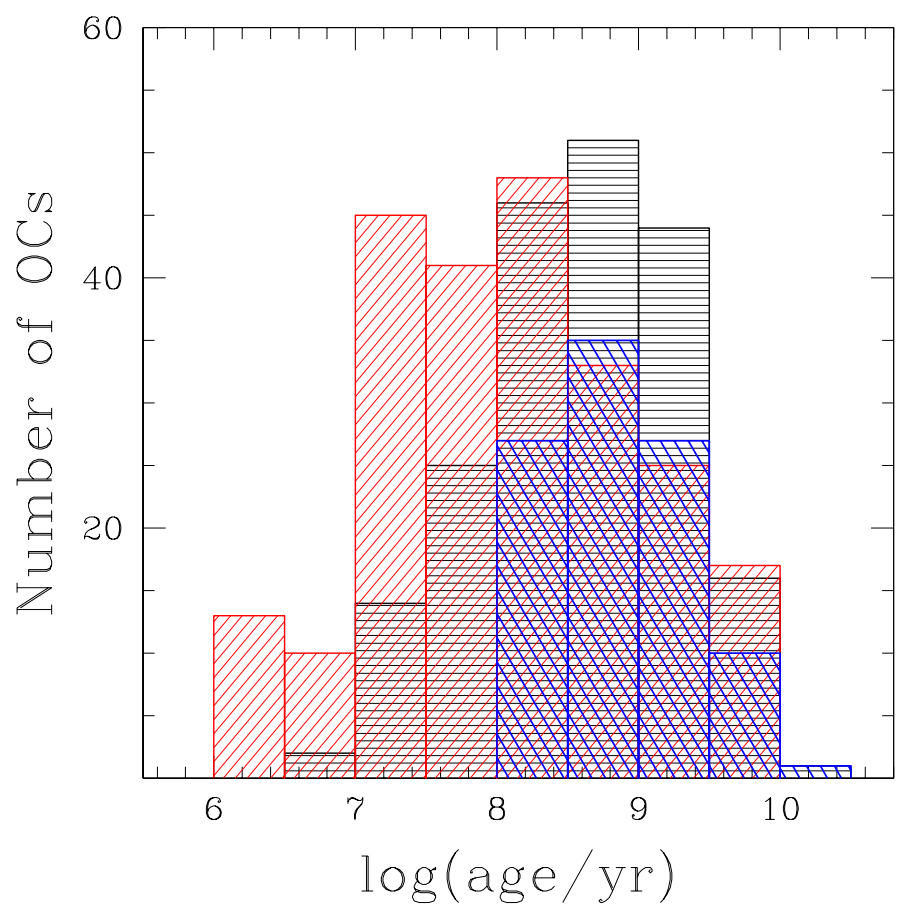

Fig. 14.5 Number distributions of Galactic OCs containing BSSs as a function of age. Horizontal shading represents the statistics from the Ahumada \& Lapasset [2] catalogue. The hatching from the bottom left to the top right represents the statistics from AL95, while that from the top left to the bottom right shows the number distribution of our working sample (100 Galactic OCs).

example, we analysed the BSS distribution functions versus the MS turn-off point, as a function of $M_{V}$ and $(B-V)$. In such a way, we can detect differences in the BSS distributions in the CMD between the AL95 and AL07 statistics. It is shown that the distribution functions of both $M_{V}$ and $(B-V)$ do not exhibit any essential differences between the AL95 and the AL07 catalogues. Therefore, we continue on the basis of the results from our previous work, i.e., the parameters of the sample clusters and the BSS population from AL95.

Details of the BSS properties are presented in Figs. 14.6 14.8 Figure 14.6 shows $N_{\mathrm{BSS}}$ as a function of the age, metallicity and richness of the sample OCs. The richness of a star cluster is represented by $N_{2}$, which is the number of cluster member stars within 2 magnitudes below the cluster's MS turn-off. The open circles represent the results for the 100 Galactic OCs. Because of the small number of member stars and even smaller number of BSSs in the individual OCs, the results directly derived from Fig. 14.6 are very stochastic, and thus we use the ratio of $N_{\mathrm{BSS}} / N_{2}$ to reduce the effects of stochasticity. We use this ratio as definition of the specific fre- 
quency of BSS components in SSPs. We calculated the standard deviation $(\sigma)$ of the ratio for the entire sample, i.e., $\sigma=\sqrt{\frac{\sum_{i=1}^{N}\left(\frac{N_{\mathrm{BSS}}}{N_{2}} i \frac{\overline{N_{\mathrm{BSS}}}}{N_{2}}\right)^{2}}{N \times(N-1)}}$ and $N=100$. We marked the OCs with $\frac{N_{\mathrm{BSS}}}{N_{2}} \leq \frac{\overline{N_{\mathrm{BSS}}}}{N_{2}}+1 \sigma$ with filled circles in Fig. 14.6

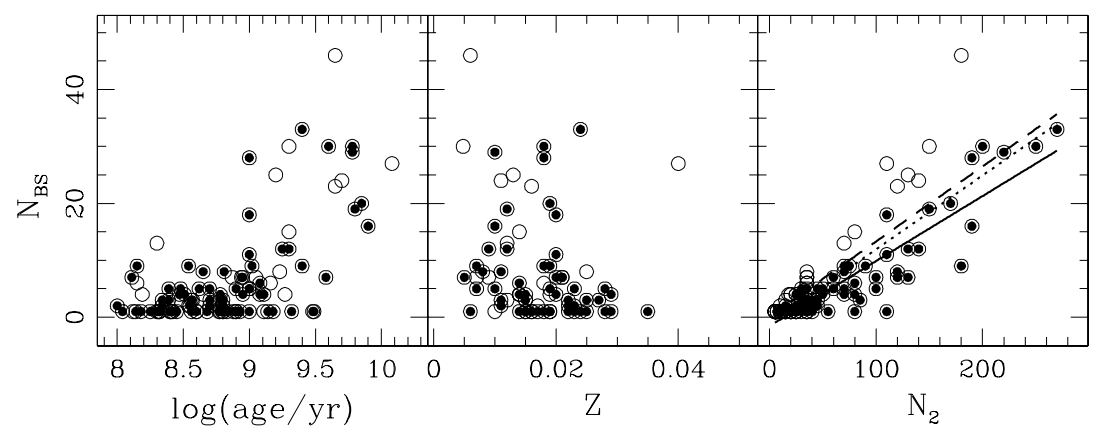

Fig. 14.6 Correlations (if any) between $N_{\mathrm{BSS}}$ and the age, metallicity and richness $\left(N_{2}\right)$ of Galactic OCs, respectively. The open circles show the results for the 100 OCs in our working sample. The filled circles mark the OCs with $N_{\mathrm{BSS}} / N_{2}$ less than the average ratio plus $1 \sigma$ (standard deviation). The solid line in the right-hand panel is the least-squares fit to the filled circles; it is given by Eq. 14.1. For reference, the dotted line in the right-hand panel is the least-squares fit to all OCs (open circles), and the dashed line is the fit to OCs with ages $\geq 1.0 \mathrm{Gyr}$.

The left-hand panel in Fig. 14.6 shows $N_{\mathrm{BSS}}$ versus cluster age on a logarithmic scale. $N_{\mathrm{BSS}}$ seems largely insensitive to age until a sudden increase for ages greater than 1.0 Gyr. Based on this figure it is hard to discern any correlation between the two parameters, and it is also risky to jump to the conclusion that $N_{\mathrm{BSS}}$ is not correlated with age. The seemingly constant $N_{\mathrm{BSS}}$ for age $<1.0 \mathrm{Gyr}$ could be caused by the confusion of defining an accurate MS turn-off point and a BSS population in relatively young star clusters. In our model construction, we have not adopted any correlation between $N_{\mathrm{BSS}}$ and age. The only correlation we used is that between $N_{\text {BSS }}$ and $N_{2}$ (shown in the right-hand panel in Fig. 14.6, see below). In fact, for SSPs, $N_{\text {BSS }}$ and age are related through the SSP's $N_{2} . N_{2}$ increases following the initial mass function (IMF) slope as the SSP ages, and so does $N_{\text {BSS }}$ through the correlation between $N_{\text {BSS }}$ and $N_{2}$.

The middle panel of Fig. 14.6 shows $N_{\text {BSS }}$ as a function of metallicity for our sample OCs with published metallicity information. No correlation can be established. We previously studied whether the BSS strengths are sensitive to metallicity. We did not find any obvious correlation between these two parameters either (see Fig. 18 in [37]).

The right-hand panel of Fig. 14.6 clearly shows that only $N_{\mathrm{BSS}}$ and $N_{2}$ are correlated. The implication of this correlation is that $N_{\mathrm{BSS}}$ is proportional to the richness 


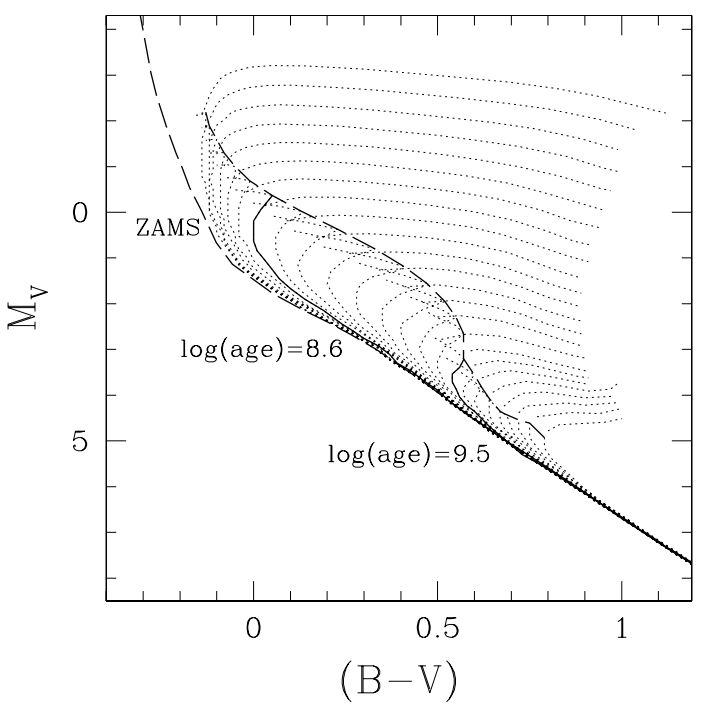

Fig. 14.7 Criteria used for our age-bin selection. The dotted lines are the isochrones for ages between 0.1 and $20 \mathrm{Gyr}$, truncated at the bottom of the RGB phase. The two dashed lines mark the MS stage between the zero-age MS (ZAMS) and terminal-age MS (TAMS). The choice of age bin is shown as the solid lines.

of an SSP, but not (at least not obviously) to any other parameter, such as age or metallicity. The solid line in the right-hand panel is the least-squares fit to the filled circles. For reference, we present two more fits for different samples. The dotted line is the least-squares fit to all OCs (open circles), while the dashed line is the fit to those OCs that are older than $1.0 \mathrm{Gyr}$. It is hard to tell which fit is most accurate. We choose the solid line for estimating $N_{\text {BSS }}$ in an SSP simply to avoid exaggeration of the BSS-enhanced intensity in our SSPs. The correlation can be empirically described as

$$
N_{\text {BSS }}=(0.114 \pm 0.006) \times N_{2}-(1.549 \pm 0.731) .
$$

The uncertainties in the coefficients result from the $1 \sigma$ uncertainty in $N_{\mathrm{BSS}} / N_{2}$.

The loci of BSSs in cluster CMDs are fixed by their formation and evolutionary processes [14], which could still be stochastic owing to the varying physical conditions among star clusters. Thus, it is impossible to construct the specific distribution function of the BSS population for each individual SSP. A feasible approach to generate a BSS population in the CMD is to work out a uniform BSS distribution function.

The dotted lines in Fig. 14.7 are isochrones with ages between 0.1 and $20 \mathrm{Gyr}$, truncated at the bottom of the RGB phase. The two dashed lines, i.e. the zero age MS (ZAMS) and the boundary between the MS and post-MS phases, highlight the entire MS stage for all isochrones. The distance between the two dashed lines be- 

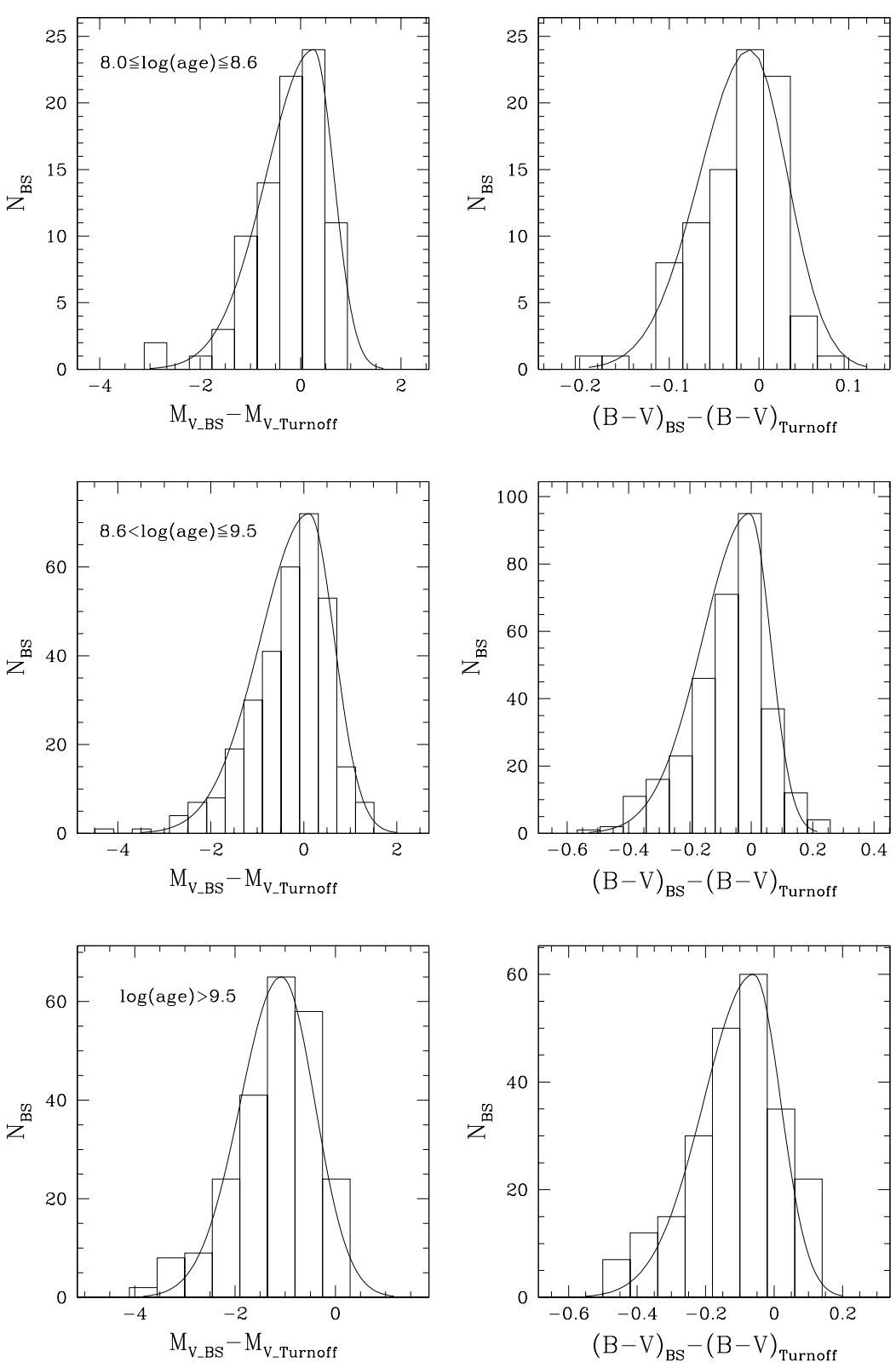

Fig. 14.8 BSS distribution functions in our OCs' CMDs in three different age bins as a function of $M_{V}$ (left panels) and the $(B-V)$ (right panels). In each panel, a gaussian profile is used to describe either side of the peak separately. 
comes narrower in the colour range in the CMD for older - $\log ($ age/yr $) \geq 9.5$ - and younger $-\log ($ age/yr $) \leq 8.6$ - SSPs, which means that a uniform BSS distribution function will certainly push a BSS population towards red colours for SSPs with a MS turn-off close to the ZAMS. To avoid uncertainties associated with the distance between the MS turn-off points and the ZAMS, we consider BSS distribution functions in three different age bins, i.e., $8.0 \leq \log ($ age/yr $) \leq 8.6,8.6<\log ($ age $/ y r) \leq 9.5$, and $\log ($ age/yr $)>9.5$. The solid lines in the figure show the age-bin selection.

Since the bin selection is done empirically, we tested that a small change in the age-bin selection, i.e., $\Delta \log ($ age $/ y r)=0.1$, cannot effectively modify the BSS distribution in the CMDs and, consequently, affect our model results. Specifically, we construct the BSS distribution functions with $\log ($ age $/ y r)=8.6$ included in the older age bin and calculate the $(U-B)$ and $(B-V)$ colours of the SSP with $Z=0.02$ and $\log ($ age $/ y r)=8.6$. The colour changes resulting from adopting different distribution functions are $<0.0001 \mathrm{mag}$ for both colours. The same result is found for a test with $\log ($ age $/ \mathrm{yr})=9.5$.

We align the MS turn-off points of all OCs in our working sample to obtain a sufficient number of BSSs for good statistics. Figure 14.8 shows the distribution functions of BSSs versus the MS turn-off point in three different age bins as a function of $M_{V}$ (left panels) and $(B-V)$ (right panels). For each of the distributions, a gaussian profile is adopted to fit either side of the peak separately, based on which we construct the BSS population in the CMD of an SSP using Monte Carlo simulations in two dimensions $\left(M_{V}\right.$ and $\left.(B-V)\right)$. All gaussian profiles have the same standard format,

$$
f(x)=\exp \left[-\frac{(x-\mu)^{2}}{2 \sigma^{2}}\right],
$$

where $\mu$ is the peak position and $\sigma$ the standard deviation. The notation $x=\Delta M_{V}=$ $M_{V_{\mathrm{BSS}}}-M_{V_{\mathrm{TO}}}$ refers to the increment in luminosity $\left(M_{V}\right)$ of a BSS with respect to the SSP's turn-off luminosity; $x=\Delta(B-V)=(B-V)_{\mathrm{BSS}}-(B-V)_{\mathrm{TO}}$ is the equivalent increment in colour index $(B-V)$. The specific values of $\mu$ and $\sigma$ for different $x$ ranges are listed in Table 14.1. Here, $f^{-}(x)$ and $f^{+}(x)$ refer to the leftand right-hand sides of the gaussian profiles, respectively.

Since the distribution functions are constructed empirically from observational statistics, the values of both $\mu$ and $\sigma$ are inevitably sensitive to the selection of the bin size. To find reasonable descriptions for the gaussian profiles, we start exploring the statistics of $M_{V}$ and $(B-V)$ in each age bin with a bin size of $\Delta M_{V}=0.20$ and $\Delta(B-V)=0.010 \mathrm{mag}$, and we subsequently increase the bin size in steps of $\Delta M_{V}=0.05$ and $\Delta(B-V)=0.005$ mag until the distribution resembles a gaussian function. The adopted bin sizes for $\Delta M_{V}$ and $\Delta(B-V)$ for each age bin are given in column (3) of Table 14.1 The $\mu$ and $\sigma$ values listed in the table are calculated based on the corresponding bin size.

In addition to the distribution function, a further boundary that also constrains BSS positions in the CMD is the ZAMS. Any BSSs located beyond the ZAMS, i.e., with bluer colours than the ZAMS for the same luminosity, will not be generated by 
Table 14.1 Gaussian profile parameters for BSS distribution functions in different age bins

\begin{tabular}{ccrccccc}
\hline $\begin{array}{c}\text { Age bin } \\
(\mathrm{yr})\end{array}$ & & $\begin{array}{r}\text { bin size } \\
(\mathrm{mag})\end{array}$ & $\begin{array}{r}\mu \\
(\mathrm{mag})\end{array}$ & $\sigma$ & $\begin{array}{c}f^{-}(x) \\
x \text { range }\end{array}$ & $\sigma$ & $\begin{array}{c}f^{+}(x) \\
x \text { range }\end{array}$ \\
\hline $8.0 \leq \log ($ age $) \leq 8.6$ & $M_{V}$ & 0.45 & 0.26 & 0.9454 & {$[-3.00,0.26]$} & 0.4056 & {$[0.26,1.65]$} \\
& $(B-V)$ & 0.03 & -0.01 & 0.0563 & {$[-0.19,-0.01]$} & 0.0418 & {$[-0.01,0.12]$} \\
$8.6<\log ($ age $) \leq 9.5$ & $M_{V}$ & 0.40 & 0.112 & 1.0464 & {$[-3.50,0.112]$} & 0.5470 & {$[0.112,2.00]$} \\
& $(B-V)$ & 0.075 & -0.005 & 0.1544 & {$[-0.530,-0.005]$} & 0.0678 & {$[-0.005,0.220]$} \\
$\log ($ age $)>9.5$ & $M_{V}$ & 0.55 & -1.08 & 0.8155 & {$[-3.83,-1.08]$} & 0.6711 & {$[-1.08,1.20]$} \\
& $(B-V)$ & 0.08 & -0.06 & 0.1472 & {$[-0.55,-0.06]$} & 0.0837 & {$[-0.06,0.20]$} \\
\hline
\end{tabular}

our program. This assumption is made mainly because we treat BSSs as MS stars and describe them using standard MS models.

Our sample of 100 Galactic OCs has limited parameter coverage in age and metallicity, i.e., it covers ages from 0.1 to $12 \mathrm{Gyr}$ and metallicities from $Z=0.0048$ to 0.035 (Xin et al. 2007, their table 1). To consider the BSS contributions for the full set of SSP models, some extrapolations of the parameter space have been adopted based on the results from OCs and some reliable working assumptions: (i) ages from 0.1 to $20 \mathrm{Gyr}$, with the lower limit set by the difficulty to identify BSS components in star clusters younger than $100 \mathrm{Myr}$, while the BSS properties are not expected to change dramatically in very old stellar populations; and (ii) metallicities $Z=0.0004,0.004,0.008,0.02$ and 0.05 . We extend the metallicity to a lower boundary of $Z=0.0004$ and a maximum value of $Z=0.05$ because the statistics of OCs show that BSS behaviour is not sensitive to metallicity. ( $Z=0.0001$ is not included in the models because horizontal branch stars, instead of BSSs, dominate the energy in the ultraviolet and blue bands in extremely metal-poor populations.)

Table 14.2 Fundamental ingredients of the BSS-SSP models

\begin{tabular}{lll}
\hline Name & Property & Source \\
\hline Padova1994 isochrones & $Z=0.0001-0.05$ Age=4Myr-20Gyr Bertelli et al. (1994) \\
BaSeL spectral library & $\begin{array}{l}91 \AA-160 \mu \mathrm{m} \\
\text { median resolution } \lambda / \Delta \lambda \approx 300\end{array}$ & Lejeune et al. (1997) \\
Initial mass function & $\begin{array}{l}\xi(\log m) \propto m^{-1.35} \\
\text { table } 1 \text { in Chabrier }(2003)\end{array}$ & $\begin{array}{l}\text { Salpeter IMF (Salpeter 1955) } \\
\text { Canonical IMF (Kroupa 2002) }\end{array}$ \\
\hline
\end{tabular}

\subsubsection{Building the Empirical SSP Library}

With the distribution functions of BSS in a population, it is now possible to build an empirical library of stellar populations. The fundamental ingredients of our models are listed in Table 14.2 . For convenience, we use the widely adopted BC03 models 
as reference. Modifications owing to BSSs are calculated as increments to the $\mathrm{BC} 03$ SSPs of the same age and metallicity. We adopted the Padova 1994 isochrones [5] and the BaSeL spectral library [20] because they homogeneously cover the widest ranges of age and metallicity, and the longest wavelength range. The Padova 2000 isochrones [15] are based on a more recent equation of state and low-temperature opacities compared to Padova 1994. However, we decided against adopting them for our model construction, because $\mathrm{BC} 03$ do not recommend to use their SSPs based on the Padova 2000 isochrones. They state that their models based on the Padova 2000 isochrones

tend to produce worse agreement with observed galaxy colours.

High-resolution observational spectral libraries (e.g., [28, 18]) suffer from problems related to limited parameter coverage. Instead of combining spectra from different libraries to enlarge our parameter coverage, we decided to use only the theoretical library.

For consistency with BC03, we adopted the Salpeter [31] and Chabrier [8] IMFs. As clearly shown by [10] the Chabrier IMF is almost indistinguishable from the Kroupa IMF [16, 17] when normalised as $\int_{0.1}^{100} \xi(m) \mathrm{d} m=1 \mathrm{M}_{\odot}$, which is exactly how both $\mathrm{BC} 03$ and we ourselves normalise the SSP models. Using such a normalisation, the slight differences between the two IMFs cannot cause any effective modifications as regards the BSS contribution to SSPs. Therefore, we refer to the IMF (in addition to the Salpeter IMF) as the "Canonical IMF" throughout this work. It can be conveniently described by a two-part power law, $\xi(m) \propto m^{-\alpha_{i}}$, with $\alpha_{1}=1.3$ for the stellar-mass range $0.08 \leq m / \mathrm{M}_{\odot}<0.5$ and $\alpha_{2}=2.3$ for $m \geq 0.5 \mathrm{M}_{\odot}$ [16], or in terms of a power law plus a lognormal form as presented in Table 1 of [8].

Our construction procedure for SSP models including BSS contributions is summarised as follows: ( $i$ ) We use the standard models (we use BC03 in this work, but we can in principle use any other SSP flavour as well) to represent the integrated spectrum of the "normal" SSP member stars. We subsequently use the statistical properties of BSSs from Galactic OCs to generate the BSS population for the appropriate SSP; and (ii) We calculate the spectrum of the BSS population and combine it with the spectrum of the normal member stars after the appropriate flux calibration. The composite spectrum is the integrated spectrum of the BSS-corrected SSP.

In detail, the model construction includes the following steps:

1) We assume that any given model SSP contains $10^{5}$ original member stars. The corresponding normalisation constant, $A$, for a given IMF is calculated as

$$
10^{5}=A \times \int_{m_{1}}^{m_{\mathrm{u}}} \phi(m) \mathrm{d} m
$$

where $\phi(m)$ is the IMF, $m_{1}=0.1 \mathrm{M}_{\odot}$ and $m_{\mathrm{u}}=100 \mathrm{M}_{\odot}$.

2) The SSP's $N_{2}$ number is calculated using

$$
N_{2}=A \times \int_{m_{1}}^{m_{2}} \phi(m) \mathrm{d} m,
$$

${ }^{1}$ see their Fig. 8 
where $m_{2}$ is the mass of the SSP's MS turn-off point and $m_{1}$ is the mass on the MS 2 mag below the turn-off point.

3) The SSP's $N_{\text {BSS }}$ then follows from Eq. 14.1.

4) The distribution of the BSS population in the CMD is generated using Monte Carlo simulations in two dimensions in the CMD, i.e., $M_{V}$ and $(B-V)$, based on the gaussian profiles described by Eq. (14.2) and Table 14.1 .
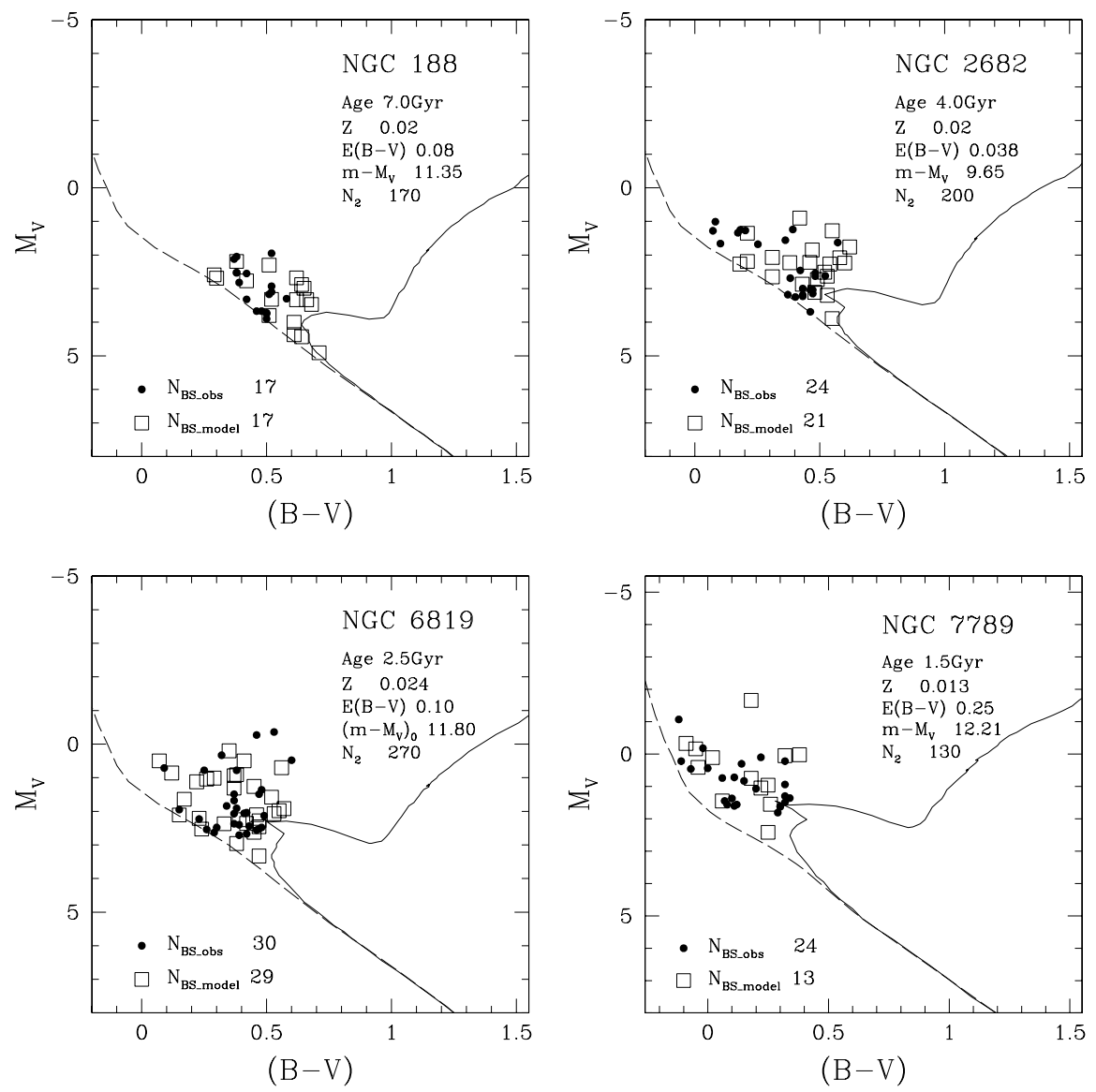

Fig. 14.9 Comparison between the observed and modelled BSS populations in the CMDs of four representative Galactic OCs. In each panel, the fundamental cluster parameters are given in the top right-hand corner, the solid curve is the isochrone representing the cluster's age and metallicity, the dashed line is the zero-age MS, the solid circles are the observed BSSs from AL95, and the open squares are the model BSSs based on Monte Carlo simulations. $N_{\text {BSS_model }}$ is calculated using the observed value of $N_{2}$ in Eq. 14.1 .

Figure 14.9 shows the comparison between the observed and modelled BSS populations in the CMDs of four representative Galactic OCs. In each panel, the funda- 
mental cluster parameters are listed in the top right-hand corner, the solid curve is the Padova 1994 isochrone for the cluster's age and metallicity, the dashed line is the ZAMS, the solid circles are the observed BSSs from AL95 and the open squares are the BSSs generated by the Monte Carlo simulation. We calculate $N_{\text {BSS_model }}$ from the observed $N_{2}$ and Eq. (14.1). Apparently, the BSS distribution fluctuates significantly for different simulations because of the small $N_{\text {BSS_model }}$ numbers. What we intend to show with this figure is that the modelled BSS population is quite reasonable and comparable with that observed.

5) Calculate the spectrum of the SSP's BSS population.

For an SSP initially consisting of $10^{5}$ stars, $N_{\mathrm{BSS}}$ always spans the range from dozens to at most hundreds of stars for ages between 0.1 and $20 \mathrm{Gyr}$, which still gives rise to stochastic fluctuations when we generate the BSS population in the CMD. These fluctuations can influence the stability of the spectrum of the BSS population. One of the best ways to reduce this stochastic effect is by using the average of a large number of models. To find the optimum number of realisations, $(i)$ we repeat the generation of BSS populations in the CMD for a given SSP 500 times (500 models) and we subsequently calculate the corresponding composite spectrum of the SSP. This implies that the 500 models yield 500 different spectra for the same SSP. (ii) We calculate the broad-band colours $(U-B),(B-V)$ and $(V-R)$ for the SSP based on the average of the spectra of a successively increasing number till 500. This calculation results in 500 values for each colour. (iii) We calculate the average of each colour using the colour values for between 200 and 500 models, thus quantifying the differences $(\delta)$ of the 500 colour values and the average colour. Finally, (iv) we identify the number of models adopted to repeat generating the BSS population in the CMD as the number for which $\delta<0.0005$ mag. Using the solarmetallicity SSPs as templates, we conclude that combining 100 models is a safe choice for SSPs characterised by different ages and IMFs.

To construct the spectrum of the model BSS population, we use Padova 1994 isochrones of the same metallicity but younger ages than the SSP to fit the position of each BSS in the CMD. We then derive the effective temperature $\left(T_{\text {eff }}\right)$ and surface gravity $(\log g)$ by interpolation between two isochrones straddling the BSS. A demonstration of this procedure is included in Fig. 14.10 The solid dots are the model BSSs, the solid line is the isochrone for the SSP's age and metallicity and the dotted lines are isochrones with ages younger than the SSP and truncated at the bottom of the RGB phase. The dashed lines are the ZAMS and the boundary of the MS and post-MS stages, respectively. In our model construction, BSSs located between the dashed lines are modelled strictly assuming that they can be represented by the MS phases of the isochrones and the remainder of the BSSs located outside the boundary are fitted with post-MS (and pre-RGB) phases.

Depending on the values of $T_{\text {eff }}$ and $\log g$, a spectrum is extracted from the Lejeune et al. [20] spectral library and assigned to the BSS. The flux of the BSS spectrum is then calibrated using the BSS's absolute magnitude and, finally, the spectrum of the BSS population is obtained by adding up all flux-calibrated BSS spectra, i.e., $F_{\mathrm{BSS}}=\sum_{i=1}^{N_{\mathrm{BSS}}} f_{\mathrm{BSS}}^{i}$. 


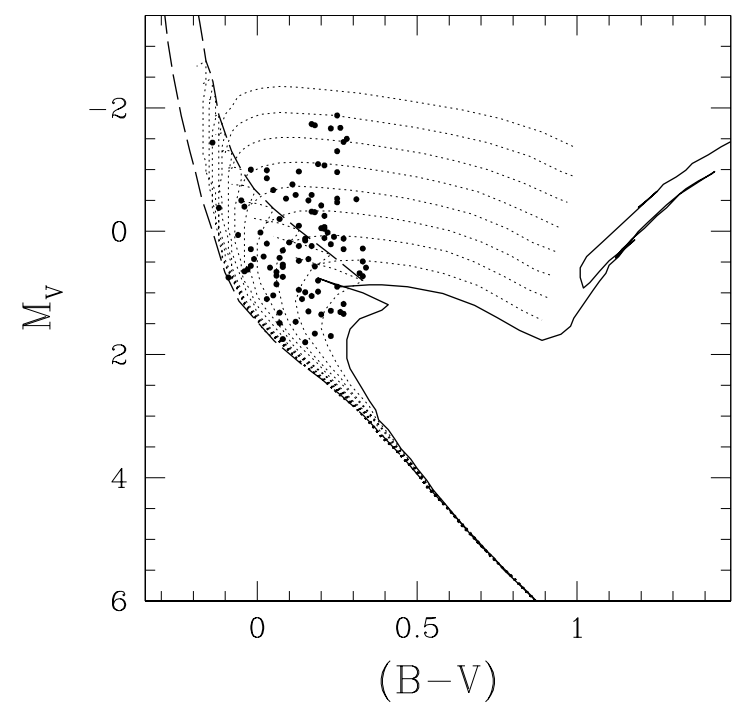

Fig. 14.10 Derivation of the basic parameters of BSSs in the CMD of a given cluster. The solid dots are the model BSSs, the solid curve is the isochrone for the SSP's age and metallicity corresponding to the cluster, and the dotted lines are isochrones representing ages younger than the SSP and truncated at the bottom of the RGB phase, that determine the fitted parameters and spectra of the BSSs. The dashed lines are the ZAMS and boundary between the MS and the end of the MS stages, respectively.

The approximation to use the spectra of single MS stars to represent the spectra of BSSs is made based on both theoretical (e.g., [3, 4]) and observational (e.g., [23, 32]) considerations. The major formation scenarios of BSSs, such as mergers of primordial binaries and dynamical encounters between stars, can replenish fresh hydrogen fuel in the core and rejuvenate BSSs to the MS stage. Liu et al. [23] studied the spectral properties of a complete sample of 24 BSSs in M67 based on spectroscopic observations with a resolution of $3.2 \AA$ pixel $^{-1}$ and covering wavelengths of 3600-6900 A. They concluded that BSS spectra can be well represented by the theoretical spectra of single stars, at least at medium resolution.

6) We use $\mathrm{BC} 03$ models to represent the spectrum of the population of normal member stars in an SSP (i.e., all member stars except the BSSs).

For a conventional SSP of age $t$ and metallicity $Z$, the integrated spectrum is given by

$$
F_{\mathrm{SSP}}(\lambda, t, Z)=B \times \int_{m_{1}}^{m_{\mathrm{u}}} \phi(m) f(\lambda, m, t, Z) \mathrm{d} m,
$$

where $\phi(m)$ is the IMF, $f(\lambda, m, t, Z)$ is the spectrum of a single star of mass $m$, age $t$ and metallicity $Z, m_{\mathrm{u}}$ and $m_{\mathrm{l}}$ are the upper and lower integration limits in mass, respectively, and ' $B$ ' is the normalisation constant required to restore the real intensity of the flux of the conventional SSP. 

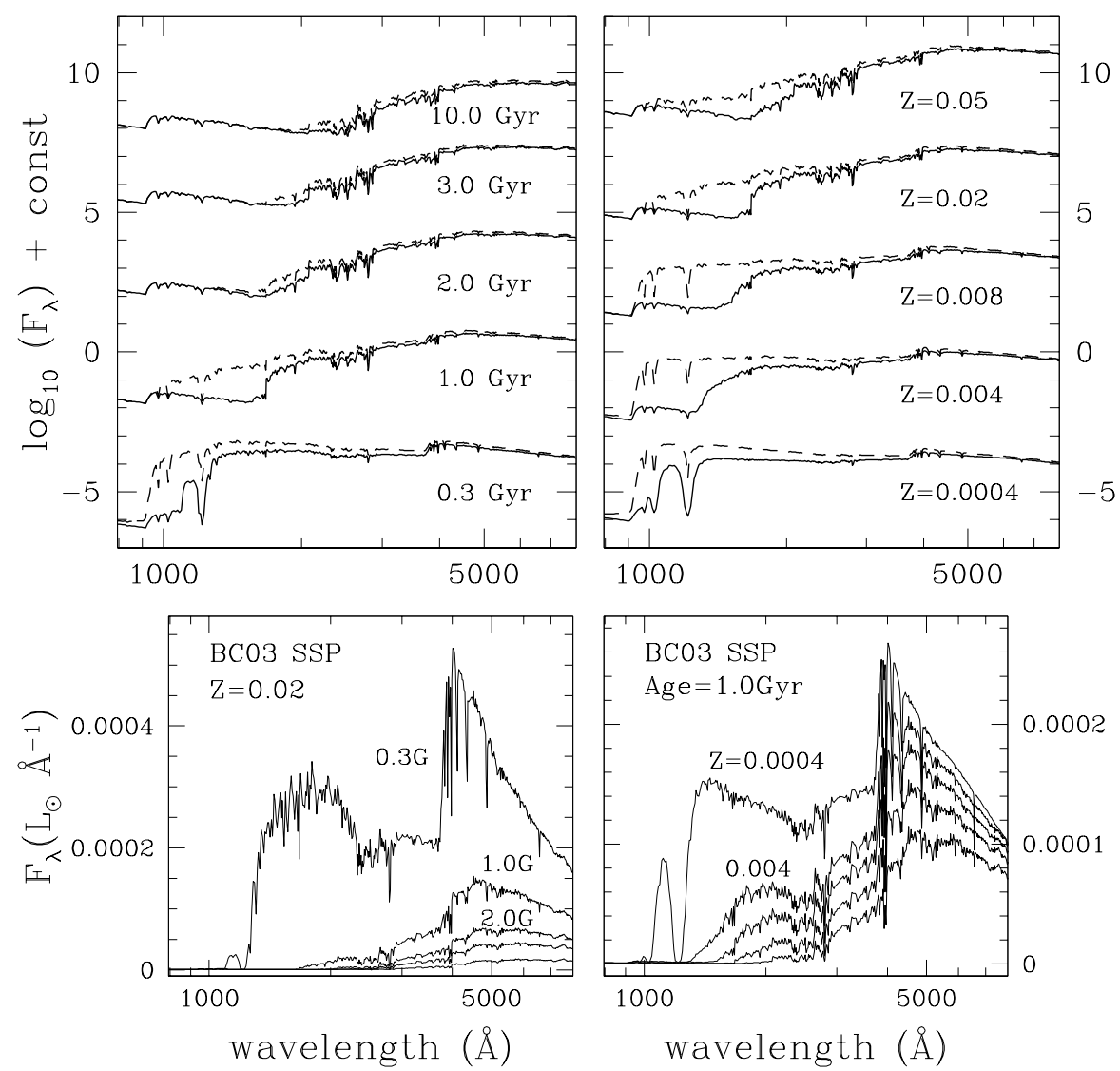

Fig. 14.11 Differences of the integrated spectral energy distributions (ISEDs) between BC03 and our models for different ages (top left-hand panel) and metallicities (top right-hand panel). The solid lines in the top panels are the BC03 ISEDs, while the dashed lines are the ISEDs resulting from our models. Because the BSS contribution is calculated as an increment to the BC03 models, the corresponding BC03 ISEDs are shown in the bottom panels for reference.

Since BC03 normalised the total mass of their model SSPs to $1 \mathrm{M}_{\odot}$ at $t=0$, ' $B$ ' is the total mass of the model SSP containing $10^{5}$ stars at $t=0$ :

$$
M_{\mathrm{tot}}=B=A \times \int_{m_{1}}^{m_{2}} \phi(m) m \mathrm{~d} m
$$

where ' $A$ ' is the normalisation constant from Eq. $(14.3), m_{1}=0.1$ and $m_{2}=100 \mathrm{M}_{\odot}$.

Similarly as for the calibration of the BSS spectra, the flux of the conventional SSP is also calibrated based on its absolute magnitude. $M_{\text {tot }}$ is used to calculate $M_{V}$ 
of the conventional SSP ( $\left.M_{V_{-} S S P}\right)$ based on Eq. (14.7), in which $M_{V-S S P(B C 03)}$ is the absolute magnitude of the corresponding $\mathrm{BC} 03 \mathrm{SSH}^{2}$ :

$$
M_{V \_S S P}=M_{V-S S P(B C 03)}+2.5 \times \log _{10}\left(1 / M_{\text {tot }}\right)
$$

7) After flux calibration, direct combination of the spectra of the BSS population and the conventional SSP yields the spectrum of the BSS-corrected SSP. As our final step, the composite spectra are normalised to the $\mathrm{BC} 03$ models by adopting the flux-calibration constant derived from Eq. (14.7) for each SSP.

As an SSP ages, the SSP's BSS population evolves to redder colours in the CMD following the movement of the SSP's MS turn-off, which implies that the "blue" in "blue stragglers" only means "bluer than the MS turn-off", not necessarily blue in colour. Since young SSPs $($ age $<0.1 \mathrm{Gyr})$ are not included in our models, and because of the presence of "yellow stragglers" in the AL95 catalogue, we decided to explore the BSS contributions to the integrated-light properties involving $U-, B$ and $V$-band energies, because they may all be significant. Specifically, we present and discuss in detail the differences in the ISEDs, broad-band colours and massto-light ratios $\left(M / L_{V}\right)$ between our models and those published by $\mathrm{BC} 03$ in this section.

Using the $Z=0.02$ models with a Salpeter IMF as example, the differences in the ISEDs between two models of different age (top left-hand panel) and metallicity (top right-hand panel) are given in Fig. 14.11. The solid lines in the top panels represent the BC03 ISEDs, while the dashed lines represent the ISEDs from our models. Since the BSS contribution is calculated as an increment to the BC03 models, the intensities of the corresponding BC03 ISEDs of different age (bottom left-hand panel) and metallicity (bottom right-hand panel) are also given in the figure.

The differences presented in the top left-hand panel show a tendency for a stronger BSS contribution for younger SSPs. BSSs have a significant effect on ISEDs for ages between 0.1 and 1.0 Gyr. A sharp enhancement appears in the UV range at $1.0 \mathrm{Gyr}$ for our models, while the UV intensity remains very low in the BC03 ISEDs. During SSP evolution, all massive single stars have left the main sequence and evolved into the red supergiant or the red giant phases in conventional SSPs of 0.1-1.0 Gyr, the UV light declines and the near-infrared intensity increases, and thus BSSs are the most luminous and bluest objects in the populations. For SSPs older than $2.0 \mathrm{Gyr}$, the intensity of the BSS contribution decreases smoothly and slowly as the population ages. The bottom left-hand panel presents the BC03 ISEDs for different ages, which show that ISEDs are stronger (brighter) for younger SSPs. This tendency is consistent with that found for the BSS contribution to ISEDs of different ages.

\footnotetext{
${ }^{2} M_{\text {tot }}$ actually quantifies the relative increase in $M_{V}$ with respect to the $M_{V}$ of an initially $1 \mathrm{M}_{\odot}$ SSP.
} 


\subsection{Discussions and Prospectives}

Fitting the spectra of unresolved galaxies using SSP models to extract information of their star formation history and current stellar contents has been a routine practice in astrophysics, but stellar populations are never simple as the current theory of stellar evolution (basically of single stars) can predict. BSSs is one of the less understood type of stars, and they are probably one of the most important contributors to the uncertainties in EPS. The other chapters in this book addressed the complicated nature of individual BSSs in both observational and theoretical aspects.

Based on observations of individual star clusters with determinations of BSSs in both numbers and photometric properties, we established a practical method to build ISED of these clusters and to use them as a constraint for the conventional SSP models and EPS analysis of galaxies. We have shown here the contents of BSSs actually altered the host stellar populations in terms of integrated light. Although there is still a very large uncertainties in the empirical treatment, our results show that EPS really should be built on more realistic SSP models that have all possible stars included properly. By analysing distribution of BSSs in a general composite CMD of all star clusters, we also developed a way to estimate BSS contributions in any stellar populations, and made a spectral library of BSS-SSP models that bares observationally constrained BSS content. The basic description of our BSS-SSP models includes:

1. The models cover the wavelength range from $91 \AA$ to $160 \mu \mathrm{m}$, ages from 0.1 to $20 \mathrm{Gyr}$ and metallicities $Z=0.0004,0.004,0.008,0.02$ (solar metallicity) and 0.05 . The metallicity $Z=0.0001$ is not included, because extended horizontal branch stars, instead of BSSs, dominate the energies in the UV and blue bands in such extremely metal-poor SSPs.

2. The models are constructed as increments to the BC03 standard SSP models using the Padova 1994 isochrones and the Lejeune et al. [20] stellar spectra. They can thus be used directly in EPS studies as replacement of BC03 for the same parameter coverage. Application of the models should be limited to the "lowresolution" regime. As each BSS spectrum is approximated by the theoretical spectrum of a single MS star, the models cannot fully account for changes in the spectral lines that are related to the formation scenarios of BSSs.

3. The essential effect of BSSs is to make an SSP's ISED hotter in the UV, blue and optical bands, and consequently turn the broadband colours much bluer. Taking the SSP models with $Z=0.02$ as an example, the differences in the broadband colours between $\mathrm{BC} 03$ and our models are $0.10 \pm 0.05 \mathrm{mag}$ in $(U-B)$, $0.08 \pm 0.02 \mathrm{mag}$ in $(B-V), 0.05 \pm 0.01 \mathrm{mag}$ in $(V-R), 0.12 \pm 0.05 \mathrm{mag}$ in $(u-g)$, $0.09 \pm 0.02 \mathrm{mag}$ in $(g-r)$ and $0.17 \pm 0.03 \mathrm{mag}$ in $(g-z)$.

Given the universal presence of BSSs in various stellar systems, the BSS-SSP models will enable the community to uncover interesting results in studies of stellar populations, although a number of limitations of the current set of model 3 The

\footnotetext{
3 available at: http://sss.bao.ac.cn/bss
} 
main source of the uncertainties in our work is true BSSs in star clusters. The current catalog of BSSs in star clusters (AL95, AL07) actually came from observations taken with different instruments at different accuracy. A survey of star clusters producing a uniform and accurate catalog is needed. An update to the catalog can be expected in a near future [12]. Also limited by small numbers of both BSSs and member stars, the statistics of BSS contribution to a cluster (population) is poor, this can be much improved by spectroscopy surveys of a substantially large number of individual stars (including BSSs) in our Galaxy, such as SDSS, LAMOST, Gaia, which can give direct census of BSSs in the Milky Way Galaxy.

Acknowledgements This work is supported by National Science Foundation of China through grants No. Y111221001 and 10973015 . We are also grateful for being invited to this ESO workshop, thanks to the organisers, in particular Giovanni Carraro and Henri Boffin.

\section{References}

1. Ahumada, J. \& Lapasset, E.: A\&AS 109, 375 (1995)

2. Ahumada, J. \& Lapasset, E.: A\&A 463, 789 (2007)

3. Benz, W. \& Hills, J.G.: ApJ 323, 614 (1987)

4. Benz, W. \& Hills, J.G.: ApJ 389, 546 (1992)

5. Bertelli, G., Bressan, A., Chiosi, C., Fagotto, F., Nasi, E.: A\&AS 106, 275 (1994)

6. Bica, E., \& Alloin, D.: A\&AS 66, 171 (1986)

7. Bruzual, G. \& Charlot, S.: MNRAS 344, 1000 (2003)

8. Chabrier, G.: PASP 115, 763 (2003)

9. Chen, X. \& Han, Z.: MNRAS 395, 1822 (2009)

10. Dabringhausen, J., Hilker, M., Kroupa, P.: MNRAS 386, 864 (2008)

11. Deng, L., Chen, R., Liu, X.S., Chen, J.S.: ApJ 524, 824 (1999)

12. Deng, L., Xin, Y. , Zhang, X. B., Li, Y.; Jiang, X.J.; Wang, G.M.; Wang, K.; Zhou, J.L.; Yan, Z.Z.; Luo, Z.Q.: IAUS, 288, 318 (2013)

13. Fan, X., Burstein, D., Chen, J.S., Zhu, J., Jiang, Z., Wu, H., Yan, H., Zheng, Z., Zhou, X. Fang, L.Z., et al.: AJ 112, 628 (1996)

14. Ferraro, F.R., Beccari, G., Dalessandro, E., Lanzoni, B., Sills, A., Rood, R.T., Pecci, F.F., Karakas, A.I., Miocchi, P., Bovinelli, S.: Nature 462, 1028 (2009)

15. Girardi, L., Bressan, A., Bertelli, G., Chiosi, C.: A\&AS 141, 371 (2000)

16. Kroupa, P.: MNRAS 322, 231 (2001)

17. Kroupa, P.: Science 295, 82 (2002)

18. Le Borgne, J.F., Bruzual, G., Pello, R., Lancon, A., Rocca-Volmerange, B., Sanahuja, B., Schaerer, D., Soubiran, C., Vilchez-Gomez, R.: A\&A 402, 433 (2003)

19. Leitherer, C., Schaerer, D., Goldader, J.D., González Delgado, R.M., Robert, C., Kune, D.F., de Mello, D.F., Devost, D., Heckman, T.M.: ApJS 123, 3 (1999)

20. Lejeune, T., Cuisinier, F., Buser, R.: A\&AS 125, 229 (1997)

21. Leonard, P.J.T. \& Linnell, A.P.: AJ 103, 1928 (1992)

22. Li, Z. \& Han, Z.: ApJ 685, 225 (2008)

23. Liu, G.Q., Deng, L., Chávez, M., Bertone, E., Davo, A.H., Mata-Chávez, M.D.: MNRAS 390, 665 (2008)

24. Mackey, M.C., Payne, M.J., Gilmore, G.F.: MNRAS 369, 921 (2006)

25. Mapelli, M., Ripamonti, E., Battaglia, G., Tolstoy, E., Irwin, M.J., Moore, B., Sigurdsson, S.: MNRAS 396, 1771 (2009)

26. Mateo, M., Hodeg, P., Schommer, R.A.: ApJ 311, 113 (1986) 
27. McCrea, W.H.: MNRAS 128, 147 (1964)

28. Pickles, A.J.: PASP 110, 863 (1998)

29. Piotto, G.: IAUS 246, 141 (2008)

30. Piotto, G., King, I.P., Djorgovski, S.G., Sosin, C., Zoccali, M., Saviane, I., De Angeli, F., Riello, M., Recio-Blanco, A., Rich, R.M., Meylan, G., Renzini, A.: A\&A 391, 945 (2002)

31. Salpeter, E.E.: ApJ 121, 161 (1955)

32. Shetrone, M.D. \& Sandquist, E.L.: AJ 120, 1913 (2000)

33. Stryker, L.L.: PASP 105, 1081 (1993)

34. Thomas, D., Maraston, C., Bender, R.: MNRAS 339, 897 (2003)

35. Vazdekis, A.: ApJ 513224 (1999)

36. Worthey, G.: ApJS 95, 107 (1994)

37. Xin, Y. \& Deng, L.: ApJ 619, 824 (2005)

38. Xin, Y., Deng, L., de Grijs, R., Kroupa, P.: MNRAS 411, 761 (2011)

39. Xin, Y., Deng, L., de Grijs, R., Mackey, A.D., Han, Z.: MNRAS 384, 410 (2008)

40. Xin, Y., Deng, L., Han, Z.: ApJ 660, 319 (2007)

41. Yi, S.K.: ApJ 582, 202 (2003) 